\title{
Multiplicity of Finite Graphs over the Real Line
}

\author{
Shosaku MATSUZAKI \\ Waseda University \\ (Communicated by K. Ota)
}

\begin{abstract}
For topological spaces $X$ and $Y$, the multiplicity $m(X: Y)$ of $X$ over $Y$ is defined by M. Gromov and K. Taniyama independently. We show that the multiplicity $m\left(G: \mathbf{R}^{1}\right)$ of a finite graph $G$ over the real line $\mathbf{R}^{1}$ is equal to the cutwidth of $G$. We give a lower bound of $m\left(G: \mathbf{R}^{1}\right)$ and determine $m\left(G: \mathbf{R}^{1}\right)$ for an $n$-constructed graph $G$.
\end{abstract}

\section{Introduction}

First, we introduce the multiplicity $m(X: Y)$ of a topological space $X$ over a topological space $Y$. Then, we explain the main purpose of this paper. We denote the cardinality of a set $A$ by \#A.

Definition 1.1. Let $X$ and $Y$ be sets and let $f: X \rightarrow Y$ be a map. For each point $y \in Y$, we define the multiplicity of $f$ at $y$, to be $\# f^{-1}(y)$. When $\# f^{-1}(y)$ is not finite, we denote $\# f^{-1}(y)=\infty$. The multiplicity $m(f)$ of $f: X \rightarrow Y$ is defined by

$$
m(f)=\sup \left\{\# f^{-1}(y) \mid y \in Y\right\} .
$$

DEFINITION 1.2 (Taniyama [8]). Let $X$ and $Y$ be topological spaces. The multiplicity $m(X: Y)$ of $X$ over $Y$ is defined by

$$
m(X: Y)=\inf \{m(f) \mid f: X \rightarrow Y \text { is a continuous map. }\} .
$$

For topological spaces $X_{1}, X_{2}, Y_{1}$ and $Y_{2}$, if $X_{1}$ is homeomorphic to $X_{2}$ and $Y_{1}$ is homeomorphic to $Y_{2}$, then $m\left(X_{1}: Y_{1}\right)=m\left(X_{2}: Y_{2}\right)$.

We note that the identical concept cardinality of $X$ over $Y$ was defined by M. Gromov [5].

In this paper we consider a graph as a topological space in the usual way.

Problem A. Given two graphs $X$ and $Y$, determine $m(X: Y)$.

We restrict our attention to the case where $X$ is a finite graph and $Y$ is an infinite graph homeomorphic to the real line $\mathbf{R}^{1}$. The vertex set of a finite graph $G$ is denoted by $V(G)$ 

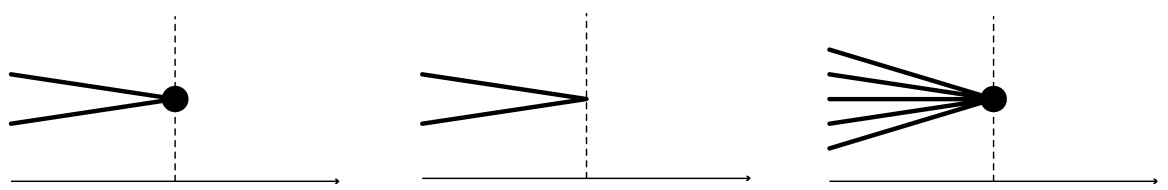

FIGURE 2.1. Examples of critical points

and the edge set of $G$ is denoted by $E(G)$. In this paper we denote $m\left(G: \mathbf{R}^{1}\right)$ by $m(G)$ for simplicity. We consider the following problem.

Problem A'. Given a finite graph $G$, determine $m(G)$.

REMARK 1.3 .

(1) For any finite graph $G, m(G)<\infty$.

(2) For any subspace $H$ of a finite graph $G, m(H) \leq m(G)$.

(3) For any subdivision $H$ of a finite graph $G, m(H)=m(G)$.

By Remark 1.3 (3) we may suppose without loss of generality that all graphs in this paper are finite and loopless. In section 2, we show that if $G$ is not edgeless, then $m(G)$ coincides with the cutwidth $c(G)$ of $G$ which is defined combinatorially in Graph theory. Then the result in [2] that the cyclic cutwidth $c c w(T)$ of a finite tree $T$ coincides with the cutwidth $c(T)$ of $T$ follows immediately by using the argument of covering space, see Note 2.3 . In section 3 , we give a lower bound of $m(G)$ for a certain graph $G$.

\section{Cutwidth and multiplicity over $\mathbf{R}^{1}$}

In this section, we introduce a special class of continuous maps that is easier to deal with than general continuous maps and then we prove that for a graph $G$ with $E(G) \neq \emptyset, m(G)$ is equal to the cutwidth $c(G)$ of $G$, a number combinatorially defined in Graph theory. This fact seems obvious but the proof requires careful consideration.

Definition 2.1. Let $G$ be a graph. A continuous map $f: G \rightarrow \mathbf{R}^{1}$ is canonical if

(a) the restriction $\left.f\right|_{V(G)}: V(G) \rightarrow \mathbf{R}^{1}$ is injective and

(b) for every edge $e \in E(G)$, the restriction $\left.f\right|_{e}: e \rightarrow \mathbf{R}^{1}$ is injective.

Given a graph $G$ and a continuous map $f: G \rightarrow \mathbf{R}^{1}$ and a point $x$ in $G, x$ is a critical point of $G$ for $f$ if there is a neighborhood $U \subset G$ of $x$ such that $f(U)$ is not a neighborhood of $f(x)$ (see Figure 2.1). We denote the set of critical points of $G$ for $f$ which are not in $V(G)$ by $C p(f)$. If $x$ is a critical point of $G$ for $f$ and $x$ is in $V(G)$, we call $x$ a critical vertex of $G$ for $f$. The following fact is a main proposition in this section.

Proposition 2.2. Let $G$ be a graph and let $f: G \rightarrow \mathbf{R}^{1}$ be a continuous map. Then, there is a canonical map $g: G \rightarrow \mathbf{R}^{1}$ such that $m(g) \leq m(f)$. 

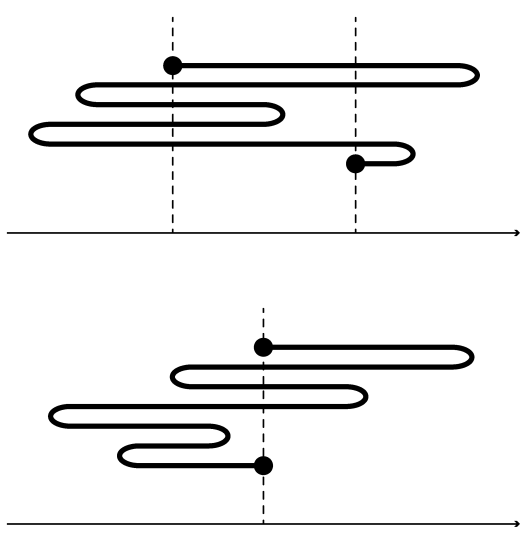

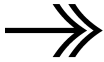

Step 1
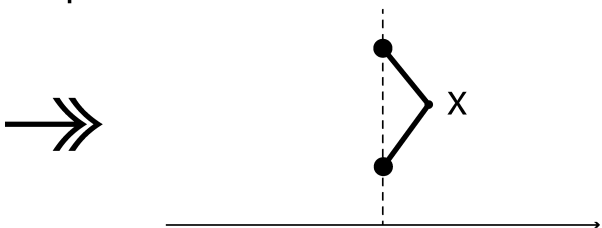

FIGURE 2.2. Step 1

Proof. Let $G$ be a graph and let $f: G \rightarrow \mathbf{R}^{1}$ be a continuous map. It is sufficient to consider the case where $G$ is connected, and for any point $y \in \mathbf{R}^{1}, \# f^{-1}(y)<\infty$. Let $V=V(G)$ and $E=E(V)$.

STEP 1. We define a new continuous map $f_{1}: G \rightarrow \mathbf{R}^{1}$ such that

(1) the restriction $\left.f_{1}\right|_{V}=\left.f\right|_{V}$,

(2) for every edge $u v \in E$ with $f(u) \neq f(v)$, the restriction $\left.f_{1}\right|_{u v}$ is injective,

(3) for every edge $u v \in E$ with $f(u)=f(v)$, both restrictions $\left.f_{1}\right|_{u x}$ and $\left.f_{1}\right|_{x v}$ are injective and $f_{1}(x)=f(x)$ where $x \in G-V$ is a point in the edge $u v$ such that $f(x) \notin \operatorname{Im}\left(\left.f\right|_{V}\right)$ (see Figure 2.2), and

(4) $\left.f_{1}\right|_{C p\left(f_{1}\right)}$ is injective.

By the construction above we have that $m\left(f_{1}\right) \leq m(f)$.

For a vertex $v \in V$, let $E_{v}$ be the set of edges that are incident to $v$.

We note here that a local perturbation may increase the multiplicity (see Figure 2.3).

Therefore we need the following Step 2-1 and Step 2-2.

STEP 2-1. Suppose that there is a critical vertex $z \in V$ for $f_{1}$ which satisfies the following condition:

(*) there exists a vertex $z^{\prime}$ such that $z^{\prime} \neq z$ and $f_{1}\left(z^{\prime}\right)=f_{1}(z)$, then we define a new continuous map $f_{1}^{\prime}: G \rightarrow \mathbf{R}^{1}$ such that

(1) for every edge $e \in E-E_{z},\left.f_{1}{ }^{\prime}\right|_{e}=\left.f_{1}\right|_{e}$, for every vertex $v \in V-z,\left.f_{1}{ }^{\prime}\right|_{v}=\left.f_{1}\right|_{v}$,

(2) $\underline{\text { Case A: }} \operatorname{Im}\left(\left.f\right|_{E_{z}}\right) \subset\left(-\infty, f_{1}(z)\right]$, $f_{1}^{\prime}(z)=f_{1}(z)-\varepsilon$, where $\varepsilon>0$ is a (sufficiently small) real number such that $\operatorname{Im}\left(\left.f_{1}\right|_{C p\left(f_{1}\right) \cup V}\right) \cap\left[f_{1}{ }^{\prime}(z), f_{1}(z)\right)=\emptyset$, 

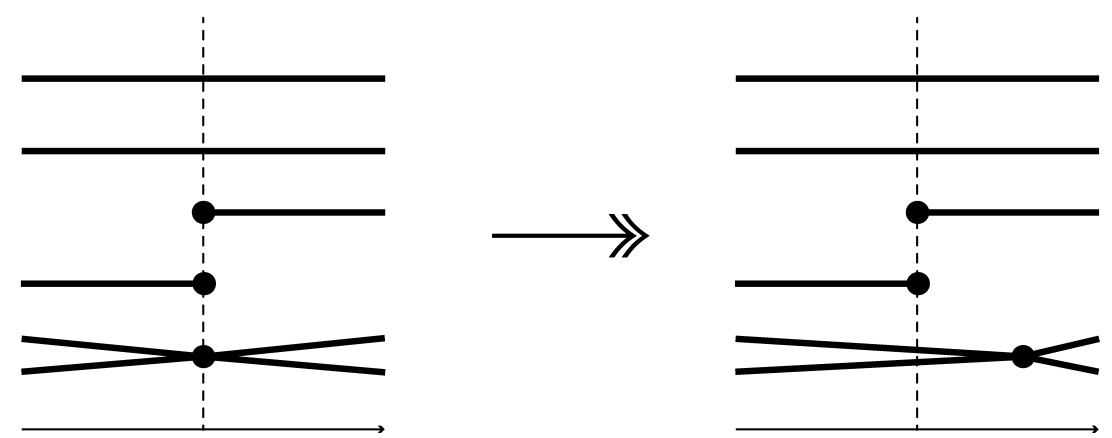

FIGURE 2.3. A local perturbation may increase the multiplicity.

Case B: $\operatorname{Im}\left(\left.f\right|_{E_{z}}\right) \subset\left[f_{1}(z),+\infty\right)$, $f_{1}^{\prime}(z)=f_{1}(z)+\varepsilon$, where $\varepsilon>0$ is a (sufficiently small) real number such that $\operatorname{Im}\left(\left.f_{1}\right|_{C p\left(f_{1}\right) \cup V}\right) \cap\left(f_{1}(z), f_{1}{ }^{\prime}(z)\right]=\emptyset$, and

(3) for every edge $e \in E_{z},\left.f_{1}{ }^{\prime}\right|_{e}$ is injective.

In Case A, we know the following facts by the construction above (see Figure 2.4).

- If $y$ is a point in $\left(-\infty, f_{1}{ }^{\prime}(z)\right) \cup\left(f_{1}(z),+\infty\right)$,

$$
\# f_{1}{ }^{-1}(y)=\# f_{1}{ }^{-1}(y) \leq m\left(f_{1}\right) \text {. }
$$

- If $y$ is a point in $\left(f_{1}{ }^{\prime}(z), f_{1}(z)\right)$,

$$
\# f_{1}{ }^{-1}(y)=\# f_{1}{ }^{-1}(y)-\# E_{z}<\# f_{1}{ }^{-1}(y) \leq m\left(f_{1}\right) .
$$

- If $y$ is a point equal to $f_{1}(z)$,

$$
\# f_{1}{ }^{-1}(y)=\# f_{1}^{-1}(y)-1<\# f_{1}^{-1}(y) \leq m\left(f_{1}\right) .
$$

- If $y$ is a point equal to $f_{1}{ }^{\prime}(z)$,

$$
\# f_{1}^{\prime}{ }^{-1}(y)=\# f_{1}^{-1}(y)-\# E_{z}+1 \leq \# f_{1}^{-1}(y) \leq m\left(f_{1}\right) .
$$

Then we have that $m\left(f_{1}^{\prime}\right) \leq m\left(f_{1}\right)$. In Case B we have the same inequality in the same way. We continue Step 2-1 until critical vertices satisfying the condition (*) disappear. A continuous map which we finally have is denoted by $f_{2}, m\left(f_{2}\right) \leq m\left(f_{1}\right)$. For a non-critical vertex $w \in V$ for $f_{2}$, we denote the set $\left\{e \in E_{w} \mid \operatorname{Im}\left(\left.f_{2}\right|_{e}\right) \subset\left(-\infty, f_{2}(w)\right]\right\}$ by $E_{w}^{-}$and the set $\left\{e \in E_{w} \mid \operatorname{Im}\left(\left.f_{2}\right|_{e}\right) \subset\left[f_{2}(w),+\infty\right)\right\}$ by $E_{w}^{+}$.

STEP 2-2. Suppose that there is a non-critical vertex $w \in V$ of $f_{2}$ which satisfies the following condition:

(**) there exists a vertex $w^{\prime}$ such that $w \neq w^{\prime}$ and $f_{2}(w)=f_{2}\left(w^{\prime}\right)$,

then we define a new continuous map $f_{2}{ }^{\prime}: G \rightarrow \mathbf{R}^{1}$ such that

(1) for every edge $e \in E-E_{w},\left.f_{2}{ }^{\prime}\right|_{e}=\left.f_{2}\right|_{e}$, for every vertex $v \in V-w,\left.f_{2}{ }^{\prime}\right|_{v}=\left.f_{2}\right|_{v}$, 

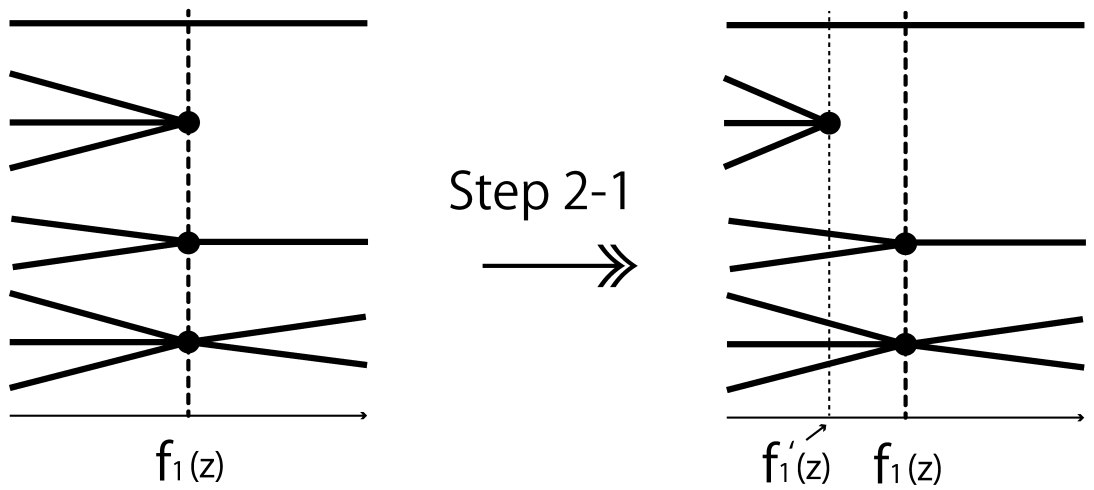

FIGURE 2.4. A local perturbation of Step 2-1

(2) Case A: $\# E_{w}^{+} \leq \# E_{w}^{-}$, $f_{2}^{\prime}(w)=f_{2}(w)-\varepsilon$, where $\varepsilon>0$ is a (sufficiently small) real number such that $\operatorname{Im}\left(\left.f_{2}\right|_{C p\left(f_{2}\right) \cup V}\right) \cap\left[f_{2}^{\prime}(w), f_{2}(w)\right)=\emptyset$,

Case B: $\# E_{w}^{+}>\# E_{w}^{-}$,

$f_{2}^{\prime}(w)=f_{2}(w)+\varepsilon$, where $\varepsilon>0$ is a (sufficiently small) real number such that $\operatorname{Im}\left(\left.f_{2}\right|_{C p\left(f_{2}\right) \cup V}\right) \cap\left(f_{2}(w), f_{2}^{\prime}(w)\right]=\emptyset$, and

(3) for every edge $e \in E_{w},\left.f_{2}{ }^{\prime}\right|_{e}$ is injective.

In Case A, we know the following facts by the construction above (see Figure 2.5).

- If $y$ is a point in $\left(-\infty, f_{2}^{\prime}(w)\right) \cup\left(f_{2}(w),+\infty\right)$,

$$
\# f_{2}{ }^{-1}(y)=\# f_{2}{ }^{-1}(y) \leq m\left(f_{2}\right) \text {. }
$$

- If $y$ is a point in $\left(f_{2}^{\prime}(w), f_{2}(w)\right)$,

$$
\# f_{2}{ }^{-1}(y)=\# f_{2}{ }^{-1}(y)-\# E_{w}^{-}+\# E_{w}^{+} \leq \# f_{2}{ }^{-1}(y) \leq m\left(f_{2}\right) .
$$

- If $y$ is a point equal to $f_{2}^{\prime}(w)$,

$$
\# f_{2}{ }^{-1}(y)=\# f_{2}{ }^{-1}(y)-\# E_{w}^{-}+1 \leq \# f_{2}^{-1}(y) \leq m\left(f_{2}\right) .
$$

- If $y$ is a point equal to $f_{2}(w)$,

$$
\# f_{2}{ }^{-1}(y)=\# f_{2}^{-1}(y)-1+\# E_{w}^{+} \text {. }
$$

The right side of the last equation may be larger than $\# f_{2}^{-1}(y)$. Consider the last case and let $y$ be the point equal to $f_{2}(w)$. Let $A \subset \mathbf{R}^{1}$ be an open neighborhood of $f_{2}(w)$ with $A \cap \operatorname{Im}\left(\left.f_{2}{ }^{\prime}\right|_{C p\left(f_{2}\right) \cup V}\right)=\left\{f_{2}(w)\right\}$. Note that $f_{2}{ }^{-1}(A)$ contains no critical vertices of $G$ for $f_{2}^{\prime}$ by Step 2-1. Therefore for any point $a \in A$ we have that $\# f_{2}^{\prime-1}(y) \leq \# f_{2}{ }^{-1}(a)$. For a point $a^{\prime} \in \mathbf{R}^{1}$ in $\left(f_{2}{ }^{\prime}(w), f_{2}(w)\right) \cap A, \# f_{2}{ }^{-1}\left(a^{\prime}\right) \leq \# f_{2}{ }^{-1}\left(a^{\prime}\right) \leq m\left(f_{2}\right)$. Thus we have that $\# f_{2}{ }^{-1}(y) \leq \# f_{2}{ }^{-1}\left(a^{\prime}\right) \leq m\left(f_{2}\right)$. Then we have that $m\left(f_{2}^{\prime}\right) \leq m\left(f_{2}\right)$. In the case B we have the same inequality in the same way. We repeat Step 2-2 until non-critical vertices satisfying 

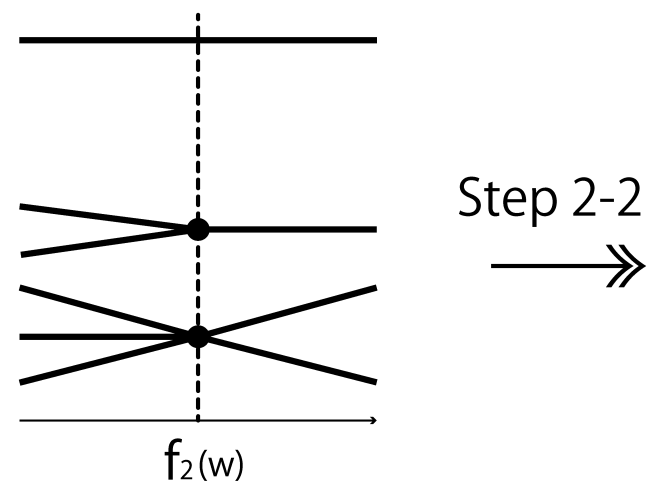

FIgURE 2.5. A local perturbation of Step 2-2. We note that there is no critical point $p \in G$ for $f_{2}$ such that $f_{2}(p)=f_{2}(w)$ at Step 2-2.

the condition $(* *)$ disappear. A continuous map which we finally have is denoted by $g$. Then $g$ is canonical and $m(g) \leq m\left(f_{2}\right) \leq m\left(f_{1}\right) \leq m(f)$ as desired.

This proposition shows that for determining the multiplicity of a graph over $\mathbf{R}^{1}$, it suffices to consider canonical continuous maps only. This assertion suggests the multiplicity of a graph $G$ with at least one edge over $\mathbf{R}^{1}$ is equal to the cutwidth of $G$. The cutwidth of a graph $G$ is defined in a combinatorial way. For a graph $G$, an injection $f: V(G) \rightarrow \mathbf{Z}$ is called a numbering of $G$. The cutwidth of a numbering $f$ is defined by $c(f)=\max _{i \in \mathbf{Z}}\left\{\#\left\{v v^{\prime} \in\right.\right.$ $E(G) \mid f(v)<i+1 / 2<f\left(v^{\prime}\right)$ or $\left.\left.f\left(v^{\prime}\right)<i+1 / 2<f(v)\right\}\right\}$. The cutwidth $c(G)$ of a graph $G$ is defined by $\min \{c(f) \mid f$ is a numbering of $G\}$ (see for example [1]). To see $m(G)=c(G)$, it is sufficient to consider the case that $G$ is a connected graph with at least one edge. For a canonical map $f: G \rightarrow \mathbf{R}^{1}$ with $m(f)=m(G)$, we may suppose that $f(V(G))$ is a subset of $\mathbf{Z}$. Then it is easy to see that $m(f)=c\left(\left.f\right|_{V(G)}\right)$. Thus we have that $m(G)=m(f)=c\left(\left.f\right|_{V(G)}\right) \geq c(G)$. The converse $m(G) \leq c(G)$ is obtained in a similar way. Thus we have that $m(G)=c(G)$. Generally, determining the cutwidth of a graph is known as one of NP-hard problems (see for example [4]).

Note 2.3. For a graph $G$, the cyclic cutwidth $\operatorname{ccw}(G)$ is defined analogously in a combinatorial way, which corresponds to $m\left(G: \mathbf{S}^{1}\right)$. See [1]. Let $T$ be a finite tree. Let $\mathbf{S}^{1}$ be a unit circle and let $f: T \rightarrow \mathbf{S}^{1}$ be a continuous map such that $m(f)=\operatorname{ccw}(T)$. Let $\phi: \mathbf{R}^{1} \rightarrow \mathbf{S}^{1}$ be a universal covering projection. Since $T$ is simply connected, there is a continuous map $\tilde{f}: T \rightarrow \mathbf{R}^{1}$ such that $f=\phi \circ \tilde{f}$. Then we have that $c(T)=m(T) \leq$ $m(\tilde{f}) \leq m(f)=c c w(T)$. Since $c c w(G) \leq c(G)$ for any $G$, we have that $c c w(T)=c(T)$. Thus we have the main result in [2]. B. Kloeckner also points out this fact in [6]. 


\section{Edge connectivity and multiplicity over $\mathbf{R}^{1}$}

For a family $\mathcal{P}$ of paths of a graph $G, \mathcal{P}$ is said to be $k$-edge-disjoint if $\# \mathcal{P}=k$ and for every pair of distinct paths $p, p^{\prime} \in \mathcal{P}, p \cap p^{\prime} \subset V(G)$. The edge-connectivity $\lambda(G)$ of a graph $G$ is $k$ if for every pair of distinct vertices $v, v^{\prime} \in V(G)$, there is a $k$-edge-disjoint family of $v$ - $v^{\prime}$ paths and there are distinct vertices $w, w^{\prime} \in V(G)$ such that there are no $(k+1)$ edge-disjoint families of $w-w^{\prime}$ paths. The multiplicity of a graph over $\mathbf{R}^{1}$ is related to the edge-connectivity of the graph. The following proposition claims that the edge-connectivity of a graph is a lower bound of the multiplicity of the graph over $\mathbf{R}^{1}$.

Proposition 3.1. Let $G$ be a graph and let $f: G \rightarrow \mathbf{R}^{1}$ be a canonical map. Then, for any point $y \in f(G)-f(V(G)), \# f^{-1}(y) \geq \lambda(G)$, therefore $m(G) \geq \lambda(G)$.

The main purpose of this paper is to give a lower bound of multiplicity for the following graphs over $\mathbf{R}^{1}$.

Definition 3.2. A connected graph $G$ is said to be $n$-constructed from graphs $H_{1}, \ldots, H_{n}$ if

(a) $H_{1}, \ldots, H_{n}$ are connected subgraphs of $G$,

(b) $G=\bigcup_{i=1}^{n} H_{i}$ and

(c) for any distinct positive integers $i, j$, the intersection $H_{i} \cap H_{j}$ is exactly one vertex of $G$.

We denote the largest integer that is not greater than $x$ by $\lfloor x\rfloor$.

There are several known lower bounds for cutwidths. See [1], [3], [6] and [7].

THEOREM 3.3. Let $G$ be a graph n-constructed from $H_{1}, H_{2}, \ldots, H_{n}$. Then,

$$
m(G) \geq \min \left\{m\left(H_{i}\right) \mid 1 \leq i \leq n\right\}+\min \left\{\lambda\left(H_{i}\right) \mid 1 \leq i \leq n\right\} \cdot\left\lfloor\frac{n-1}{2}\right\rfloor .
$$

For the proof of Theorem 3.3, we need the following lemma, whose proof is obvious.
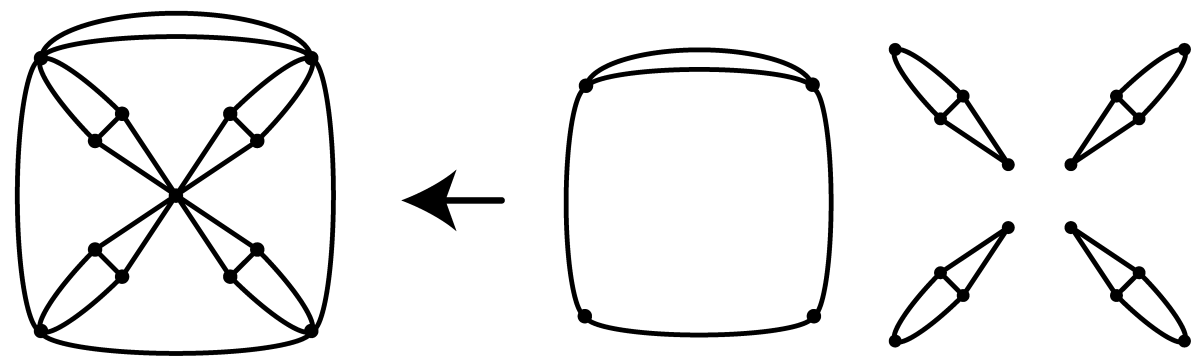

FIGURE 3.1. The left is a 5-constructed graph from the five mutually homeomorphic graphs on the right. 
LEMMA 3.4. Let $G$ be a graph with $\# E(G) \geq 1$ and let $f: G \rightarrow \mathbf{R}^{1}$ be a canonical map. Then, there is an open interval $I \subset \operatorname{Im}(f) \subset \mathbf{R}^{1}$ such that for any point $y \in I$, $\# f^{-1}(y) \geq m(G)$.

Proof OF THEOREM 3.3. The prototype of the following proof can be seen in [5]. The proof is given by an induction on $n$. When $n$ is equal to 1 , the claim is true. We assume that the claim is true for odd positive integer $k \geq 1$. Let $G$ be a graph $(k+2)$-constructed from $H_{1}, \ldots, H_{k+2}$, and let $f: G \rightarrow \mathbf{R}^{1}$ be a canonical map. Since $G$ is connected, there are distinct vertices $x, y \in V(G)$ such that $\operatorname{Im}(f)=[f(x), f(y)] \subseteq \mathbf{R}^{1}$. We may suppose without loss of generality that $x$ and $y$ are contained in the union $H_{k+1} \cup H_{k+2}$. Now, $\bigcup_{i=1}^{k} H_{i}$ is denoted by $G^{\prime}$ and $H_{k+1} \cup H_{k+2}$ is denoted by $G^{\prime \prime}$.

Since $\lambda\left(G^{\prime \prime}\right)=\min \left\{\lambda\left(H_{k+1}\right), \lambda\left(H_{k+2}\right)\right\}$, by Proposition 3.1, for any point $z \in$ $\operatorname{Im}\left(\left.f\right|_{G^{\prime \prime}}\right)-\operatorname{Im}\left(\left.f\right|_{V\left(G^{\prime \prime}\right)}\right)$,

$\left.\# f\right|_{G^{\prime \prime}} ^{-1}(z) \geq \lambda\left(G^{\prime \prime}\right)=\min \left\{\lambda\left(H_{k+1}\right), \lambda\left(H_{k+2}\right)\right\}$.

By Lemma 3.4 and the assumption of the induction, there exists an open interval $I \subset$ $\operatorname{Im}\left(\left.f\right|_{G^{\prime}}\right)$ such that for any point $w \in I,\left.\# f\right|_{G^{\prime}}{ }^{-1}(w) \geq m\left(G^{\prime}\right) \geq \min \left\{m\left(H_{i}\right) \mid 1 \leq i \leq\right.$ $k\}+\min \left\{\lambda\left(H_{i}\right) \mid 1 \leq i \leq k\right\} \cdot\left\lfloor\frac{k-1}{2}\right\rfloor$.

Therefore we have that for any point $w^{\prime} \in I-f\left(V\left(G^{\prime \prime}\right)\right)$,

$$
\begin{aligned}
m(G) \geq & \# f^{-1}\left(w^{\prime}\right) \geq m\left(G^{\prime}\right)+\lambda\left(G^{\prime \prime}\right) \\
\geq & \min \left\{m\left(H_{i}\right) \mid 1 \leq i \leq k\right\}+\min \left\{\lambda\left(H_{i}\right) \mid 1 \leq i \leq k\right\} \cdot\left\lfloor\frac{k-1}{2}\right\rfloor+ \\
& \quad \min \left\{\lambda\left(H_{k+1}\right), \lambda\left(H_{k+2}\right)\right\} \\
\geq & \min \left\{m\left(H_{i}\right) \mid 1 \leq i \leq k+2\right\}+ \\
& \quad \min \left\{\lambda\left(H_{i}\right) \mid 1 \leq i \leq k+2\right\} \cdot\left\lfloor\frac{(k+1)-1}{2}\right\rfloor .
\end{aligned}
$$

Therefore for positive odd integer $n$, the claim is true.

Next we prove the case that $n>0$ is even. For a positive even integer $n$, let $G$ be an $n$-constructed graph from $H_{1}, \ldots, H_{n}$. Since $G$ contains an $(n-1)$-constructed graph $\bigcup_{i=1}^{n-1} H_{i}$ as a subgraph and $\left\lfloor\frac{n-1}{2}\right\rfloor=\left\lfloor\frac{n-2}{2}\right\rfloor$, we have the following from the assumption of the induction:

$$
\begin{aligned}
& m(G) \geq m\left(\bigcup_{i=1}^{n-1} H_{i}\right) \\
& \geq \min \left\{m\left(H_{i}\right) \mid 1 \leq i \leq n-1\right\}+\min \left\{\lambda\left(H_{i}\right) \mid 1 \leq i \leq n-1\right\} \cdot\left\lfloor\frac{(n-1)-1}{2}\right\rfloor \\
& \geq \min \left\{m\left(H_{i}\right) \mid 1 \leq i \leq n\right\}+\min \left\{\lambda\left(H_{i}\right) \mid 1 \leq i \leq n\right\} \cdot\left\lfloor\frac{n-1}{2}\right\rfloor .
\end{aligned}
$$

Thus, for any positive integer $n$, the claim is true.

By using Theorem 3.3, lower bounds of the multiplicity of the following graphs in Figure 3.2, Figure 3.3, and Figure 3.4 are obtained. Since we can easily make continuous maps which realizes the lower bounds of the multiplicities of those graphs, we know that these bounds are equal to the multiplicities of these graphs. Moreover, we can prove that every graph in Figure 


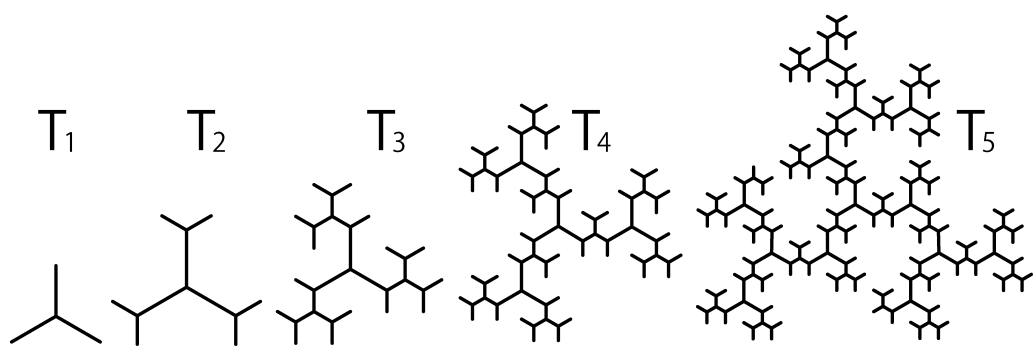

FIGURE 3.2. $m\left(T_{n}\right)=n+1 . T_{i+1}$ is a 3 -constructed graph from three copies of $T_{i}$. $\lambda\left(T_{i}\right)=1$.

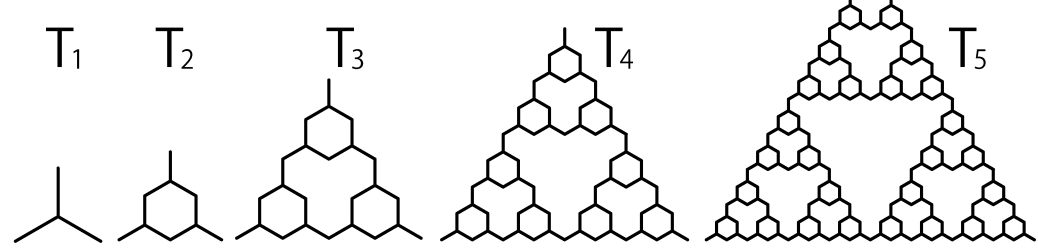

FIGURE 3.3. $m\left(T_{n}\right)=n+1, T_{i+1}$ is a 3 -constructed graph from three copies of $T_{i}$. $\lambda\left(T_{i}\right)=1$.
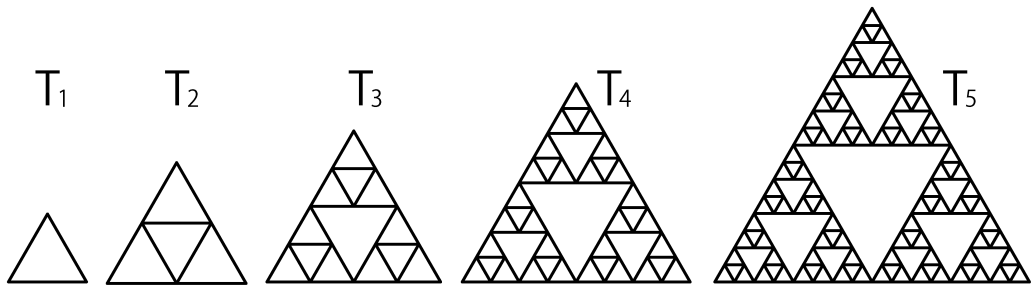

FIGURE 3.4. $m\left(T_{n}\right)=2 n . T_{i+1}$ is a 3-constructed graph from three copies of $T_{i}$. $\lambda\left(T_{i}\right)=2$.

3.2 is critical with respect to the multiplicity. Namely, the multiplicity of any proper subgraph is strictly less than the multiplicity of the graph. We also note here that a lower bound of the multiplicity of the graph in Figure 3.1 is 7 . In fact, It is known that the multiplicity of the graph is just 7 .

ACKnOwledgments. The author is most grateful to Professors Shin'ichi Suzuki and Kouki Taniyama. The author is also grateful to the referee for his/her helpful comments. 


\title{
References
}

[ 1 ] D. Barth, F. Pellegrini, A. Raspaud and J. Roman, On bandwidth, cutwidth, and quotient graphs, Informatique Théorique et Applications, 29, No. 6 (1995), 487-508.

[2] J. D. Chavez and R. TRApP, The cyclic cutwidth of trees, Discrete Applied Mathematics, 87 (1998), $25-32$.

[ 3 ] F. R. K. Chung and P. D. SEymour, Graphs with small bandwidth and cutwidth, Discrete Mathematics, 75 (1989), 113-119.

[ 4 ] F. GaVRIL, Some NP-complete problem on graphs, Proc. 1977 Conf. on Inf. Sc. and Syst., Johns Hopkins University (1977), 91-95.

[ 5 ] M. Gromov, Singularities, expanders and topology of maps. Part 2: From combinatorics to topology via algebraic isoperimetry, Geometric and Functional Analysis, 20 (2010), 416-526.

[6] B. Kloeckner, Cutwidth and degeneracy of graphs, arXiv:0907.5138, (2010).

[ 7 ] Y. LiN, X. Li and A. YANG, A degree sequence method for the cutwidth problem of graphs, Applied Mathematics-A Journal of Chinese Universities, 17, Issue 2 (2002), 125-134.

[ 8 ] K. TANiYAma, Multiplicity of a space over another space, J. Math. Soc. Japan, 64, No. 3 (2012), 823-849.

\author{
Present Address: \\ GRADUATE SCHOOL OF EDUCATION, \\ WASEDA UNIVERSITY, \\ Nishi-WASEDA 1-6-1, SHINJUKU-KU, TOKYO, 169-8050 JAPAN. \\ e-mail: shosaku@ruri.waseda.jp
}

